\title{
Calidad de vida general y podológica en mujeres posmenopáusicas con alteraciones del metabolismo óseo: estudio observacional
}

\author{
General and podiatric quality of life in postmenopausal women with bone metabolism \\ disorders: observational study

\begin{abstract}
Antonio Córdoba-Fernández ${ }^{1}$, Luz María Alonso-Barbero y y María José Montoya-García
'Departamento de Podología. Facultad de Enfermería, Fisioterapia y Podología, Universidad de Sevilla. Sevilla, España. ${ }^{2}$ Departamento de Medicina. Facultad de Medicina. Universidad de Sevilla. Sevilla, España
\end{abstract}

Palabras clave:

Menopausia,

osteopenia,

osteoporosis, calidad de

vida, salud podológica.
Keywords:

Menopause, osteopenia osteoporosis, quality of life, podiatric health.
Recibido: 22-01-2020

Aceptado: 01-04-2020

\section{Resumen}

Introducción: Durante la menopausia se producen cambios fisiológicos que pueden traducirse en una merma importante de la calidad de vida general de las mujeres. El objetivo del presente estudio fue conocer la calidad de vida general y podológica, así como el riesgo de fractura a diez años vista en una muestra de mujeres menopáusicas diagnosticadas de osteopenia u osteoporosis.

Pacientes y métodos: En este estudio observacional descriptivo y transversal se administró el cuestionario FootHealth Status (FHS) validado y adaptado al castellano a 40 mujeres menopáusicas diagnosticadas de osteopenia (T-score $\leq-1.0$ y $>-2.5$ ) o de osteoporosis (T-score $\leq-2.5$ ) mediante densitometría. Para determinar el riesgo de fractura mayor osteoporótica y fractura de cadera se empleó la herramienta Frax ${ }^{\oplus}$.

Resultados: Existe una tendencia negativa en ambos grupos respecto a la percepción de salud general y podológica. Las pruebas de contraste mostraron diferencias significativas respecto a la percepción de salud general entre los grupos, siendo menor en el grupo de osteopenia $(p<0.048$ ). El riesgo de sufrir fractura mayor o fractura de cadera fue significativamente mayor en el grupo de mujeres con osteoporosis $(p=0.003)$

Conclusiones: La pérdida de masa ósea propia de la menopausia parece estar asociada a una merma en la calidad de vida general y podológica de las mujeres. No obstante, esta puede estar condicionada también por otros factores asociados al climaterio lo que explica que esta relación haya sido más fuerte en las mujeres con osteopenia. El riesgo de fractura mayor osteoporótica o fractura de cadera fue significativamente mayor en las mujeres con osteoporosis establecida.

\section{Abstract}

Introduction: During menopause, physiological changes occur that can result in a significant decrease in the general quality of life of women. The aim of the present study was to know the general and podiatric quality of life as well as to determine the risk of fracture at ten years seen in a sample of menopausal women diagnosed with osteopenia or osteoporosis.

Patients and methods: In this descriptive and cross-sectional observational study, the FootHealth Status (FHS) questionnaire validated and adapted to Spanish was administered to 40 menopausal women diagnosed with osteopenia (T-score $<-1.0$ ), or osteoporosis (T-score $\leq-2.5)$ by densitometry $(\mathrm{DXA})$. The Frax ${ }^{\circledR}$ tool was used to determine the risk of major osteoporotic fracture and hip fracture.

Results: There was a negative trend in both groups regarding the perception of general and podiatric health. The contrast tests showed significant differences regarding the general health perception among the groups being lower in the osteopenia group $(p<0.048)$. The risk of major fracture or hip fracture was significantly higher in the group of women with osteoporosis $(p=0.003)$.

Conclusions: The loss of bone mass typical of menopause seems to be associated with a decrease in the general and podiatric quality of life of women. However, this also can to be conditioned by other factors associated with the climacteric, which explains why this relationship has been stronger in women with osteopenia. The risk of major osteoporotic fracture or hip fracture was significantly higher in women with established osteoporosis. 


\section{INTRODUCCIÓN}

La menopausia es una etapa fisiológica de transición a una nueva situación biológica que lleva aparejada, además de la pérdida de la capacidad reproductiva, una serie de manifestaciones clínicas que repercuten tanto en la esfera física como anímica de la mujer y que pueden tener un impacto negativo en su calidad de vida ${ }^{1}$. La prevalencia de síntomas relacionados con dolor muscular y articular en mujeres menopáusicas son variados y difieren según el área geográfica y el estado posmenopáusico, aunque cada vez más se consideran un problema de salud pública por la merma en la calidad de vida que pueden originar. No obstante, la calidad de vida después de la menopausia está influenciada por otros muchos factores no menopáusicos y los resultados observados tienen una alta variabilidad en función del país y del estado posmenopáu$\operatorname{sico}^{2,3}$. Los estudios que relacionan la clínica asociada a la menopausia con los cambios que esta origina en la calidad de vida de las mujeres son escasos, y aunque la asociación entre dolores musculoesqueléticos y deficiencia de estrógenos parece fuerte, aún falta un vínculo causal entre ambos ${ }^{4}$.

Por otro lado, la guía Europea para el diagnóstico y manejo de la osteoporosis (2019) establece que la presencia de una baja masa ósea (osteoporosis) por debajo de valores de densidad ósea mineral (DMO) de -2.5 medidos mediante DXA, no debe considerarse categoría de enfermedad sino como riesgo de fractura, y solo debe ser tenida en cuenta a efectos de descripción epidemiológica ${ }^{5}$.

Los estudios que han analizado la calidad de vida en mujeres posmenopáusicas con osteoporosis han mostrado que, independientemente de la presencia de otras patologías, esta se ve significativamente disminuida y confirman que esta disminución es mayor en mujeres con fractura osteoporótica previa ${ }^{6,7}$. Por otro lado, pese a la alta prevalencia de patología podológica en mujeres menopáusicas, los estudios existentes llevados a cabo con escalas validadas para analizar el impacto de estos sobre la calidad de vida son escasos ${ }^{8,9}$. La escasa evidencia disponible pone de manifiesto la necesidad de analizar la forma en la que la menopausia puede afectar a la calidad de vida en general y la podológica en particular de las mujeres, especialmente aquellas con alteraciones del metabolismo óseo. La realización de estudios a partir de los cuales los profesionales sanitarios puedan poner en marcha estrategias de salud encaminadas a mejorar la calidad de vida de las mujeres menopáusicas se antoja esencial, y con este objetivo nos planteamos el presente estudio observacional para analizar la calidad de vida general y podológica en una muestra de mujeres menopáusicas, con o sin alteraciones del metabolismo óseo.

\section{PACIENTES Y MÉTODOS}

\section{Diseño del estudio y sujetos}

El presente trabajo es un estudio observacional descriptivo y transversal Ilevado a cabo en la Unidad de Osteoporosis del Servicio de Medicina Interna del Hospital Universitario Virgen Macarena de Sevilla (España). La muestra se obtuvo a partir de mujeres posmenopáusicas de entre 50-70 años de raza blanca caucásica del área de influencia del Hospital Universitario Virgen Macarena (Sevilla). Las mujeres premenopáusicas dentro de la franja etaria con densidad ósea mineral (DMO) dentro de la normalidad o mujeres con osteoporosis secundaria fueron excluidas. Entre mayo y septiembre de 2018 se seleccionaron 40 mujeres posmenopáusicas que habían sido estudiadas de forma consecutiva para análisis de la DOM, de columna lumbar (L1-L4), cuello de fémur y cadera total, mediante Absorciometría Dual de Rayos X (DXA, Hologic-discovery, HologicINc, Waltham, Massachussetts, EE. UU.). Las mujeres seleccionadas fueron divididas en dos grupos en función del grado de afectación. Un grupo de 20 mujeres diagnosticadas de osteopenia (T-score $\leq-1.0$ y > -2.5) en cualquiera de las localizaciones señaladas y otro grupo de 20 mujeres diagnosticadas de osteoporosis (T-score $\leq-2.5$ ), en al menos alguna de las tres localizaciones estudiadas. El estudio fue aprobado por el Comité de Ética de la Investigación Biomédica de la Junta de Andalucía (Cl:0724-N-09). A todas las participantes se les facilitó información y firmaron el preceptivo consentimiento informado, donde se recogía el objetivo del estudio y cómo se iban a gestionar sus datos. Seguidamente se administró el cuestionario FHSQ (Foot Health Status Questionaire) adaptado al castellano. Las diferentes categorías obtenidas a partir del cuestionario y su equivalencia numérica fueron recopiladas empleando un programa Excel). Para el análisis descriptivo de las variables se agruparon algunos dominios del cuestionario. Para el análisis de la variable "dolor en los pies", se agruparon las preguntas 1, 2, 3 y 4 del cuestionario en un único ítem evaluando el tipo, severidad y duración del dolor en los pies en cada uno de los grupos. Del mismo modo, para el análisis de la variable función del pie se agruparon las preguntas 5, 6, 7 y 8 del cuestionario de modo que evaluamos esa variable en términos de impacto sobre la función física del pie. Para el análisis de la variable salud general del pie se agruparon las preguntas 9 y 13 del cuestionario donde se determina una valoración de la imagen corporal relativa de los pies, y por último la variable dificultad para calzarse (preguntas 10, 11 y 12 del cuestionario) nos permitió evaluar el estilo de vida relacionado con el calzado y los pies. Para el análisis de la calidad de vida general en cada uno de los grupos se prefijaron cuatro dominios agrupando las variables salud general (pregunta 14 y 19), actividad física (pregunta 15), capacidad social (pregunta 16 y 18) y vitalidad (pregunta 17).

Mediante la herramienta online Frax ${ }^{\circledR}$, versión española (https://www.fundacionmf.org.ar/visor-producto.php?cod_producto=3535), se calculó la probabilidad a 10 años de fractura mayor osteoporótica (FMO) y fractura de cadera (FC). Del mismo modo se recopilaron otras variables como edad, altura, peso, DMO y T-score, así como datos relativos a hábitos higiénico-conductuales (consumo de tabaco y alcohol), 
episodios previos de fractura y presencia de otras patologías asociadas.

Para evaluar la microarquitectura ósea de las mujeres estudiadas se empleó el software TBS (Trabecular Bone Score) que evaluó la tasa promedio de variación local en escala de grises de la intensidad de cada pixel de la imagen en 2D obtenida de la DXA.

\section{Análisis estadístico}

Para el análisis descriptivo se calcularon las frecuencias absolutas, frecuencias relativas, valores medios, desviación típica, valores mínimo, máximo y mediana. Una vez planteadas las hipótesis estadísticas sobre las variables a estudiar se analizaron las relaciones entre dichas variables teniendo en cuenta un nivel de confianza del $95 \%$. Para llevar a cabo el contraste de hipótesis, y en función del comportamiento de las variables, se emplearon según el caso pruebas paramétricas o no paramétricas para muestras independientes. En todos los casos se consideró un nivel de significación $p<0.05$. Se realizó también un análisis correlacional entre variables para establecer la intensidad y tendencia en la relación entre dos variables. El análisis estadístico se llevó cabo mediante el paquete estadístico SPSS versión 22.0 para Windows (IBM Corp., Armonk, Nueva York, EE. UU.). En este estudio se aplicaron las siguientes pruebas: prueba T-Student para muestras independientes, prueba U de Mann-Whitney, ANOVA de un factor y prueba de Kruskal-Wallis. Para el análisis de las variables cualitativas, hemos utilizado el test Chi cuadrado para ver si existe algún tipo de relación (dependencia) entre las variables, a través de las tablas cruzadas. Se realizó también un análisis correlacional para indicar la intensidad y tendencia en la relación entre dos variables cuantitativas. Se utilizó el coeficiente de correlación de Pearson cuando las dos variables cumplieron la condición de normalidad, en caso contrario, se calculó el coeficiente de correlación de Spearman (Rho).

\section{RESULTADOS}

Se incluyeron inicialmente 60 mujeres a las que se les había realizado densitometría ósea (DMO) durante el 2018, de ellas 12 presentaron valores DMO dentro de la normalidad y 8 denegaron incluirse en el estudio. Finalmente se incluyeron 40 mujeres con una edad media de $63.28 \pm 4.70$, de las cuales la mitad presentaron valores compatibles con osteopenia y la otra mitad con osteoporosis. La distribución de las variables fue normal para la altura, peso, DMO y T-score para el grupo de osteopenia y para la altura, peso, DMO, T-score y FMO en el grupo de osteoporosis. Ambos grupos fueron comparables en cuanto a edad, peso y estilo de vida. Respecto a la calidad de vida general y podológica las pruebas de contraste mostraron diferencias significativas respecto a la percepción de salud general entre los grupos con una tendencia negativa en el grupo de osteopenia con respecto al de osteoporosis. Las preguntas del cuestionario donde las pruebas de contraste evidenciaron una diferencia significativa entre los grupos correspondieron a las preguntas 14, 15 y 19 (Tabla I).

La DMO media en el grupo de osteopenia fue ligeramente superior a la media registrada en el grupo de osteoporosis sin diferencias significativas $(0.79 \pm 0.13$ frente a $0.78 \pm 0.13$ ). Respecto a T-Score, se observó un valor promedio disminuido en el grupo de osteoporosis frente al grupo de osteopenia sin diferencias significativas $(-1.47 \pm 1.22$ frente a $-1.59 \pm 0.76)$. Respecto al riesgo de fractura en 10 años, se observaron diferencias significativas para las variables FMO y FC, siendo el grupo con osteoporosis el que mostró una probabilidad mayor de fracturas a los 10 años (Tabla II).

Para ambos grupos, la prueba Rho de Spearman mostró una correlación directa entre el riesgo de FC y FMO. Del mismo modo, el test mostró una correlación inversa entre el riesgo de fractura y los valores de T-score y DMO en ambos grupos, siendo esta mayor en el grupo de osteoporosis (Tablas III y IV).

\begin{tabular}{|c|c|c|c|c|}
\hline & & $\begin{array}{l}\text { Osteopenia } \\
20(50 \%)\end{array}$ & $\begin{array}{l}\text { Osteoporosis } \\
20(50 \%)\end{array}$ & Significación \\
\hline \multirow{3}{*}{ P14 En general usted diría que su salud es: } & Muy buena & $0(0,0 \%)$ & $2(10,0 \%)$ & \multirow{3}{*}{0,048} \\
\hline & Aceptable & $8(40,0 \%)$ & $13(65,0 \%)$ & \\
\hline & Mala & $12(60,0 \%)$ & $5(25,0 \%)$ & \\
\hline \multirow{3}{*}{$\begin{array}{l}\text { P15 ¿Cuánto le limita coger o llevar una bolsa de la } \\
\text { compra? }\end{array}$} & Nada & $9(45,0 \%)$ & $17(85,0 \%)$ & \multirow{3}{*}{0,026} \\
\hline & Un poco & $6(30,0 \%)$ & $1(5,0 \%)$ & \\
\hline & Regular & $5(25,0 \%)$ & $2(10,0 \%)$ & \\
\hline \multirow{3}{*}{$\begin{array}{l}\text { P19A Creo que me pongo enferma más fácilmente } \\
\text { que otras personas }\end{array}$} & Verdadero & $6(30,0 \%)$ & $2(10,0 \%)$ & \multirow{3}{*}{0,035} \\
\hline & No lo sé & $3(15,0 \%)$ & $0(0,0 \%)$ & \\
\hline & Falso & $11(55,0 \%)$ & $18(90,0 \%)$ & \\
\hline
\end{tabular}


Tabla II. Características antropométricas de la muestra estudiada.

\begin{tabular}{llll} 
& Osteopenia & Osteoporosis & p-valor \\
\hline Edad (años) & $64.32 \pm 4$ & $62.24 \pm 5$ & $>0.05$ \\
\hline Altura $(\mathrm{cm})$ & $155.94 \pm 5.51$ & $153.24 \pm 7.09$ & $>0.05$ \\
\hline Peso $(\mathrm{kg})$ & $70.71 \pm 9.60$ & $62.24 \pm 9.83$ & $>0.05$ \\
\hline IMC $\left(\mathrm{kg} / \mathrm{m}^{2}\right)$ & $29.46 \pm 2.4$ & $26.59 \pm 3.6$ & $>0.05$ \\
\hline Riesgo de FMO $(\%)$ & $6.13 \pm 3.69$ & $8.18 \pm 3.09$ & $<0.05$ \\
\hline Riesgo de FC $(\%)$ & $0.40 \pm 0.41$ & $0.75 \pm 0.69$ & $<0.05$ \\
\hline DMO $\left(\mathrm{gHA} / \mathrm{cm}^{2}\right)$ CF & $0.79 \pm 0.13$ & $0.78 \pm 0.13$ & $>0.05$ \\
\hline TBS & $-1.59 \pm 0.76$ & $-1.47 \pm 1.22$ & $>0.05$ \\
\hline
\end{tabular}

IMC: índice de masa corporal. FMO: fractura mayor osteoporótica. FC: fractura de cadera. DMO: densidad mineral ósea. CF: cuello fémur. TBS: trabecular bone score.

Tabla III. Correlación entre las variables FC y FMO en cada grupo.

\begin{tabular}{|c|c|c|c|c|}
\hline & & & & FMO \\
\hline \multirow{6}{*}{ Rho de Spearman } & \multirow{3}{*}{ OSTEOPENIA } & \multirow{3}{*}{ FC } & Coeficiente de correlación & 0.382 \\
\hline & & & Sig. (bilateral) & 0.097 \\
\hline & & & $\mathrm{N}$ & 20 \\
\hline & \multirow{3}{*}{ OSTEOPOROSIS } & \multirow{3}{*}{ FC } & Coeficiente de correlación & $0.626^{* *}$ \\
\hline & & & Sig. (bilateral) & 0.003 \\
\hline & & & $\mathrm{N}$ & 20 \\
\hline
\end{tabular}

FC: fractura de cadera.

Tabla IV. Correlación entre el T-Score y el riesgo de FMO en cada grupo.

\begin{tabular}{|c|c|c|c|c|}
\hline & & & & FMO \\
\hline \multirow{6}{*}{ Rho de Spearman } & \multirow{3}{*}{ OSTEOPENIA } & \multirow{3}{*}{ T-Score } & Coeficiente de correlación & $-0.634^{* x}$ \\
\hline & & & Sig. (bilateral) & 0.003 \\
\hline & & & $\mathrm{N}$ & 20 \\
\hline & \multirow{3}{*}{ OSTEOPOROSIS } & \multirow{3}{*}{ T-Score } & Coeficiente de correlación & -0.705 \\
\hline & & & Sig. (bilateral) & 0.001 \\
\hline & & & $\mathrm{N}$ & 20 \\
\hline
\end{tabular}

\section{DISCUSIÓN}

En España, la osteoporosis afecta a aproximadamente a un $15 \%$ de la población femenina, con una prevalencia del $26 \%$ en mujeres mayores de 50 años $^{10}$. Pese a ello, son escasos los estudios que han analizado la influencia de la osteoporosis en la calidad de vida en general de mujeres menopáusicas de nuestro país y son escasos los que hayan valorado la calidad de vida podológica en estas mujeres. La mayoría de las publicaciones en este sentido ponen de manifiesto que en mujeres menopáusicas, la osteoporosis produce una merma importante en la calidad de vida de las mujeres y que esta pérdida es considerablemente mayor en mujeres con episodios previos de fractura ${ }^{3}$. Sin embargo, los escasos estudios que han analizado la calidad de vida en mujeres menopáusicas españolas por un lado, y la alta variabilidad de factores de riesgo entre diferentes poblaciones con alteraciones de la DMO, hacen que la extrapolación de datos sea difícil a la hora de evaluar la calidad de vida de las mismas ${ }^{11}$.

Existen diferentes cuestionarios de salud validados transculturalmente al castellano que, como el empleado en este estudio, han mostrado ser herramientas válidas, fiables, sensibles y fáciles de administrar para evaluar la calidad de vida $^{12,13}$. La mayoría de los estudios desarrollados en nuestro país utilizando diferentes cuestionarios ponen de manifiesto que la osteoporosis es la causa de dolor más intenso de todas las patologías asociadas a dolor crónico no oncológico, y que existe una relación directa entre la intensidad del dolor y el grado de afectación de la calidad de vida de los pacientes ${ }^{14}$. Del mismo modo, respecto a la salud podológica y calidad de 
vida, los estudios desarrollados en la población española han mostrado que la presencia de anomalías podológicas reduce sensiblemente la calidad de vida tanto en su componente físico como mental y que la calidad de vida general y podológica en mujeres menopáusicas fue significativamente menor ${ }^{8,9}$. En este estudio piloto, nuestros resultados también han mostrado una tendencia negativa respecto a la percepción de la salud general y específica podológica en ambos grupos de mujeres menopáusicas, si bien es cierto que la calidad de vida registrada en el grupo de mujeres con osteopenia ha sido significativamente menor respecto a la detectada en mujeres con osteoporosis. Esta circunstancia puede haberse debido a que, para la inclusión de las mujeres en ambos grupos, se tuvieron en cuenta los resultados obtenidos de DMO y T-Score pese a que las pruebas de contraste no mostraron diferencias significativas para ninguna de estas variables entre los grupos. Esto explicaría que las diferencias observadas respecto a la calidad de vida se han podido ver condicionadas por otras variables. La literatura que aborda la calidad de vida en la menopausia pone de manifiesto que esta se ve condicionada por múltiples aspectos relacionados con los cambios físicos y emocionales secundarios a los trastornos hormonales y no solo con la pérdida de masa ósea ${ }^{2,3}$.

Otros estudios desarrollados en nuestro medio han detectado que en mujeres osteoporóticas la calidad de vida fue más baja que en mujeres sanas para todas las dimensiones evaluadas ${ }^{15}$. En el presente estudio, dado el reducido número de mujeres inicialmente reclutado sin alteraciones del metabolismo óseo, declinamos incluir un grupo control de mujeres que nos hubiera permitido establecer de forma más precisa la influencia de las alteraciones del metabolismo óseo en su calidad de vida. En esta misma línea, y teniendo en cuenta las limitaciones del presente trabajo, nuestros resultados muestran una tendencia negativa respecto a la percepción de la salud general y podológica en ambos grupos, que curiosamente ha sido mayor en el grupo de osteopenia. Pensamos que esto puede haberse debido de una parte, a que las diferencias entre los grupos no han sido significativas respecto a los valores de DMO y T-score y de otro, a que aunque la osteopenia representa un menor grado de afectación de la calidad ósea, el complejo sintomático asociado a otros factores de riesgo como el IMC (mayor en el grupo de osteopenia) o el porcentaje de fracturas previas (mayor en el grupo de mujeres con osteopenia), pueden haber resultado determinantes.

Aunque la relación entre el dolor musculoesquelético y menopausia parece ser alta, lo cierto es que no parece existir un vínculo causal entre la deficiencia de estrógenos y el dolor musculoesquelético ${ }^{4}$. Lo que sí parece estar claro con la evidencia disponible es que la pérdida de la calidad de vida en mujeres con osteoporosis se ve incrementada cuando estas sufren algún tipo de fractura ${ }^{6}$. Con la herramienta FRA $X^{\circledR}$ hemos detectado un riesgo significativamente mayor en el grupo de osteoporosis, tanto para la FMO como para la FC. Sin embargo, los valores promedio de DMO y T-Score no difirieron significativamente entre los grupos. Recientemente se ha demostrado que el T-score tiene un valor predictivo para la fractura independientemente de la probabilidad de fractura obtenida usando el algoritmo $F R A X^{\circledR}$ y no está claro cómo el cambio en el T-score se relaciona con el riesgo de fractura ${ }^{16}$. Si bien la herramienta puede ser utilizada tanto si se cuenta con los valores T-score y DMO como si no, una fortaleza de nuestro estudio viene condicionada por el hecho de que la fiabilidad de la herramienta se ha visto incrementada al haber contado con estas dos variables para cuantificar el riesgo. La herramienta FRAX ${ }^{\circledR}$ resulta útil a la hora de tomar decisiones en base fundamentalmente a las características particulares de las pacientes, y sobre todo en base al riesgo de fractura que por otro lado no ha sido claramente establecido en nuestro país. En nuestro entorno europeo más próximo, concretamente en Reino Unido, este riesgo se ha estimado un $7 \%$, por lo que nuestro grupo de osteoporosis superaría este umbral. Hemos encontrado un riesgo absoluto de FMO al reportado por otros autores en mujeres osteoporóticas de nuestro país en el medio extrahospitalario ${ }^{15}$. Este resultado es similar al obtenido en nuestro grupo de mujeres con osteoporosis y pensamos que estas diferencias han podido deberse fundamentalmente al promedio de edad más bajo de las mujeres de nuestro estudio, ya que las guías existentes consideran pacientes de alto riesgo a las mujeres con una DMO baja, mayores de 65 años y con antecedentes maternos de $\mathrm{FC}^{17}$.

En base a los resultados preliminares del presente trabajo podemos afirmar que existe una tendencia negativa respecto a la percepción de la salud general y específica del pie en ambos grupos. El riesgo de fractura a diez años ha sido significativamente mayor en el grupo de osteoporosis. Si bien el riesgo se ha calculado a nivel de cadera, este puede ser extrapolado a otras localizaciones como el riesgo de sufrir fracturas por estrés en el pie especialmente en metatarsianos con resistencia elástica alterada. La literatura existente muestra que las fracturas de estrés en los metatarsianos tienen una alta prevalencia y suponen un $38 \%$ de todas las fracturas de estrés de la extremidad inferior, afectando mayoritariamente al segundo y tercer metatarsiano ${ }^{18}$.

Respecto a otros factores de riesgo asociados a fractura, como el IMC por debajo de los estándares considerados normales, parece ser el mejor predictor de disminución de la DMO en las mujeres ancianas. Por el contrario, es conocido que los individuos con un IMC elevado tienen mejor microarquitectura ósea que los individuos con normopeso ${ }^{19}$. Las mujeres estudiadas en nuestro grupo de osteoporosis se encuentran dentro de la categoría de sobrepeso, estando el de osteopenia muy próximo a la obesidad lo que podría explicar que el riesgo de fractura haya sido menor en este grupo.

La utilización conjunta de cuestionarios junto con la herramienta FRAX ${ }^{\circledast}$ pueden ser muy útiles en la consulta de podología para detectar el riesgo de fractura, especialmente en mujeres menopáusicas. La detección precoz del riesgo de fracturas en mujeres menopáusicas por parte 
del podólogo/a resulta esencial en el diagnóstico diferencial de metatarsalgias. Coincidimos con otros autores en la importancia de la actuación conjunta de profesionales de distintas especialidades sanitarias para no solo prevenir de forma precoz los riesgos de fractura asociados a osteoporosis, sino también abordar la resolución más rápida completa y satisfactoria de las mismas ${ }^{20}$. Con estas herramientas nos podemos anticipar a las complicaciones que puedan hacer que la calidad de vida de las mujeres menopáusicas pueda verse comprometida.

\section{CONFLICTO DE INTERESES}

Los autores no declaran conflictos de intereses relevantes con este artículo.

\section{FINANCIACIÓN}

Los autores no han recibido financiación alguna para la realización del presente estudio.

\section{BIBLIOGRAFÍA}

1. Palacios S, Neyro JL, Fernández de Cabo S, Chaves J, Rejas J. Impact of osteoporosis and bone fracture on health-related quality of life in postmenopausal women. Climateric 2013;17(1):1-11. DOI: 10.3109/13697137.2013.808182.

2. Schneider HPG, Birkhauser M. Quality of life in climacteric women. Climateric 2017;20(3):187-94. DOI: 10.1080/13697137.2017.1279599.

3. Paternina A, Monterrosa A, Romero I. Evaluación de la calidad de vida en la menopausia, mediante la escala "Menopause Rating Scale" (MRS): una revisión sistemática. Med Unab. 2011;13(3):139-45.

4. Watt FE. Musculoskeletal pain and menopause. Post Reprod Health 2018;24(1):34-43. DOI: 10.1177/2053369118757537.

5. Kanis JA, Cooper C, Rizzoli R, Reginster JY. Executive summary of the European guidance for the diagnostic and management of osteoporosis in postmenopausal women. Calcif Tissue Int. 2019;104(3):235-8. DOI: 10.1007/s00223-018-00512-x.

6. Si L, Winzenberg TM, de Graaff B, Palmer AJ. A systematic review and meta-analysis of utility-based quality of life for osteoporosis-related conditions. Osteoporos Int. 2014;25(8):1987-97. DOI: 10.1007/ s00198-014-2636-2.

7. Kanazawa I, Takeno A, Tanaka KI, Yamane Y, Sugimoto T. Osteoporosis and vertebral fracture are associated with deterioration of activities of daily living and quality of life in patients with type 2 diabetes mellitus.
J Bone Miner Metab 2019;37(3):503-11. DOI: 10.1007/s00774-0180948-6.

8. Pita S, González C, Seoane T, Pertega S, Perez S, López B. Podiatric Medical Abnormalities in a Random Population Sample 40 Years or Older in Spain. J Am Pod Med Assoc. 2014;4(6):574-82. DOI: 10.7547/87507315-104.6.574.

9. López D, López N, Losa ME, Rodríguez D, Palomo P, Becerro de Bengoa R. Impact on foot health related quality of life in a sample of menopausal women: an observational case-control study. Climacteric. 2016;19(5):501-5. DOI: 10.1080/13697137.2016.1198314.

10. Álvarez Sanz C, Rapado A, Díaz Curiel M, García JJ, Carrasco Honorato JL, Pérez Cano R. Prevalencia de osteoporosis determinada por densitometría en la población femenina española. Med Clin (Barc). 2001;116(3):86-8. DOI: 10.1016/s0025-7753(01)71732-0.

11. Ferrer J, Neyro JL, Estevez A. Identification of risk factors for prevention and early diagnosis of asymptomatic post-menopausal women. Maturitas 2005;52(Suppl 1):S7-22. DOI: 10.1016/j.maturitas.2005.06.017.

12. Sirera MJ, Sáez P, Sanz MD. Traducción y adaptación transcultural al castellano y al valenciano del Foot Health Status Questionnaire. Rev Ortop Traumatol. 2010;54(4):211-9. DOI: 10.1016/j.recot.2010.02.006.

13. Cuesta-Vargas A, Bennett P, Jimenez-Cebrian AM, Labajos-Manzanares MT. The psychometric properties of the Spanish version of the Foot Health Status Questionnaire. Qual Life Res. 2013;22(7):1739-43. DOI: 10.1007/s11136-012-0287-3.

14. Casals M, Samper D. Epidemiología, prevalencia y calidad de vida del dolor crónico no oncológico. Estudio ITACA. Rev Soc Esp Dolor. 2004; 11(5):260-9.

15. Sánchez M, Vicente MP, Martín A, Vicente E, Vicente JL. Calidad de vida en pacientes osteoporóticos en atención primaria. Actualidades en Psicología 2011;25(2):57-73.

16. Harvey N, Glüer C, Binkley N, McCloskey E, Brandi ML, Cooper C, et al. Trabecular bone score (TBS) as a new complementary approach for osteoporosis evaluation in clinical practice. Bone. 2015;78(1):216-24. DOI: 10.1016/j.bone.2015.05.016.

17. Pérez Edo L, Alonso A, Roig D, García A, Guañabens N, Peris P, et al. Actualización 2011 del Consenso Sociedad Española de Reumatología de Osteoporosis. Reumatol Clin. 2011;7(6):357-79. DOI: 10.1016/j. reuma.2011.05.013.

18. Wilson E, Kaz F. Stress fractures. Radiology an analysis of 250 consecutive cases. Radiology. 1969;92(3):481-6. DOI: 10.1148/92.3.481.

19. Giner M, Montoya MJ, Miranda C, Vázquez MA, Miranda MJ, Pérez-Cano R. Influencia de la obesidad sobre la microarquitectura y las propiedades biomecánicas en pacientes con fractura de cadera. Rev Osteoporos Metab Miner. 2017;9(1):20-7. DOI: 10.4321/S1889836X2017000100004

20. Mesa Ramos M. Prevención interdisciplinar de la fractura de cadera (editorial). Rev Osteoporos Metab Miner. 2013;5(1):5-6. DOI: 10.4321/ S1889-836X2013000100001. 